TRANSACTIONS OF THE

AMERICAN MATHEMATICAL SOCIETY

Volume 355, Number 10, Pages 4093-4110

S 0002-9947(03)03249-5

Article electronically published on June 24, 2003

\title{
BURGHELEA-FRIEDLANDER-KAPPELER'S GLUING FORMULA FOR THE ZETA-DETERMINANT AND ITS APPLICATIONS TO THE ADIABATIC DECOMPOSITIONS OF THE ZETA-DETERMINANT AND THE ANALYTIC TORSION
}

\author{
YOONWEON LEE
}

\begin{abstract}
The gluing formula of the zeta-determinant of a Laplacian given by Burghelea, Friedlander and Kappeler contains an unknown constant. In this paper we compute this constant to complete the formula under an assumption that the product structure is given near the boundary. As applications of this result, we prove the adiabatic decomposition theorems of the zeta-determinant of a Laplacian with respect to the Dirichlet and Neumann boundary conditions and of the analytic torsion with respect to the absolute and relative boundary conditions.
\end{abstract}

\section{INTRODUCTION}

In [3, Burghelea, Friedlander and Kappeler established a gluing formula for the zeta determinant of an elliptic operator on a compact manifold. This formula contains an unknown constant which can be expressed in terms of the zero coefficients of some asymptotic expansions. In this paper we compute this constant in the case when the product structure is given near the boundary, and then we apply this result to prove the adiabatic decomposition theorems for the zeta determinant and the analytic torsion. Some results of this paper are known from the work of Klimek and Wojciechowski in [6], but our method is completely different from theirs.

Let $M$ be a compact oriented $m$-dimensional manifold with boundary $Z$ ( $Z$ may be empty), and $Y$ a hypersurface of $M$ such that $M-Y$ has two components and $Y \cap Z=\emptyset$. We denote by $M_{1}, M_{2}$ the closure of each component, i.e. $M=M_{1} \cup_{Y}$ $M_{2}$. Choose a collar neighborhood $N$ of $Y$, which is diffeomorphic to $[-1,1] \times Y$, $N \cap Z=\emptyset$, and choose a metric $g$ on $M$ that is a product metric on $N$. Suppose that $E \rightarrow M$ is a complex vector bundle such that $\left.E\right|_{N}$ has the product structure, which means that $\left.E\right|_{N}=\left.p^{*} E\right|_{Y}$, where $p:[-1,1] \times Y \rightarrow Y$ is the canonical projection. Let $\Delta_{M}$ be a Laplacian acting on smooth sections of $E$, and let $\Delta_{M_{1}}, \Delta_{M_{2}}$ be the restrictions of $\Delta_{M}$ to $M_{1}$ and $M_{2}$. By a Laplacian we mean a positive semi-definite 2nd order differential operator whose principal symbol is $\sigma_{L}\left(\Delta_{M}\right)(x, \xi)=\|\xi\|^{2}$. We

Received by the editors April 15, 2002 and, in revised form, October 10, 2002.

2000 Mathematics Subject Classification. Primary 58J52, 58J50.

Key words and phrases. Zeta-determinant, gluing formula, Laplacian, Dirichlet (Neumann) boundary condition, absolute (relative) boundary condition, adiabatic decomposition.

The author was partially supported by Korea Research Foundation Grant KRF-2000-015DP0045. 
assume that $\Delta_{M}$ is $-\partial_{u}^{2}+\Delta_{Y}$ on $N$, where $\partial_{u}$ is the unit normal vector field to $Y$ on $N$, outward to $M_{1}$, and $\Delta_{Y}$ is a Laplacian on $Y$.

We denote by $D, B$ the Dirichlet boundary conditions on $Z, Y$ and by $C$ the Neumann boundary condition on $Y$, defined as follows:

$$
\begin{array}{cll}
D: C^{\infty}\left(M_{i}\right) \rightarrow C^{\infty}\left(Z \cap M_{i}\right) & \text { by } & \multicolumn{1}{c}{D(\phi)=\left.\phi\right|_{Z},} \\
B: C^{\infty}\left(M_{i}\right) \rightarrow C^{\infty}(Y) & \text { by } & B(\phi)=\left.\phi\right|_{Y}, \\
C: C^{\infty}\left(M_{i}\right) \rightarrow C^{\infty}(Y) & \text { by } & C(\phi)=\left.\left(\partial_{u} \phi\right)\right|_{Y} .
\end{array}
$$

Then the Laplacian $\Delta_{M, D}\left(\Delta_{M_{i}, B, D}, \Delta_{M_{i}, C, D}\right)$ with the Dirichlet condition on $Z$ (the Dirichlet condition on $Y$ and $Z$, the Neumann condition on $Y$ and the Dirichlet condition on $Z)$ is defined by the same operator $\Delta_{M}\left(\Delta_{M_{i}}\right)$ with domains as follows:

$$
\begin{aligned}
\operatorname{Dom}\left(\Delta_{M, D}\right) & =\left\{\phi \in C^{\infty}(M) \mid D(\phi)=0\right\}, \\
\operatorname{Dom}\left(\Delta_{M_{i}, B, D}\right) & =\left\{\phi \in C^{\infty}\left(M_{i}\right) \mid B(\phi)=0, D(\phi)=0\right\}, \\
\operatorname{Dom}\left(\Delta_{M_{i}, C, D}\right) & =\left\{\phi \in C^{\infty}\left(M_{i}\right) \mid C(\phi)=0, D(\phi)=0\right\} .
\end{aligned}
$$

For computational reasons, we consider $\Delta_{M, D_{m}}^{m}+t^{m}, \Delta_{M_{i}, B_{m}, D_{m}}^{m}+t^{m}$ and $\Delta_{M_{i}, C_{m}, D_{m}}^{m}+t^{m}\left(t \in \mathbb{R}^{+}\right)$rather than $\Delta_{M, D}, \Delta_{M_{i}, B, D}$ and $\Delta_{M_{i}, C, D}$, where $D_{m}$, $B_{m}$ and $C_{m}$ are the Dirichlet and the Neumann boundary conditions corresponding to $\Delta_{M}^{m}, \Delta_{M_{i}}^{m}$ (or $\left.\Delta_{M}^{m}+t^{m}, \Delta_{M_{i}}^{m}+t^{m}\right)$ defined as follows:

$$
\begin{aligned}
& D_{m}=\left(D, D \Delta_{M}, \cdots, D \Delta_{M}^{m-1}\right), \\
& B_{m}=\left(B, B \Delta_{M_{i}}, \cdots, B \Delta_{M_{i}}^{m-1}\right), \\
& C_{m}=\left(C, C \Delta_{M_{i}}, \cdots, C \Delta_{M_{i}}^{m-1}\right) .
\end{aligned}
$$

Note that

$$
\Delta_{M, D}^{m}+t^{m}= \begin{cases}\prod_{k=-\left[\frac{m}{2}\right]}^{\left[\frac{m-1}{2}\right]}\left(\Delta_{M, D}+e^{i \frac{2 k \pi}{m}} t\right) & \text { if } m \text { is odd } \\ \prod_{k=-\left[\frac{m}{2}\right]}^{\left[\frac{m-1}{2}\right]}\left(\Delta_{M, D}+e^{i \frac{(2 k+1) \pi}{m}} t\right) & \text { if } m \text { is even. }\end{cases}
$$

For $-\left[\frac{m}{2}\right] \leq k \leq\left[\frac{m-1}{2}\right]$, let $\alpha_{k}=e^{i \frac{2 k \pi}{m}}$ if $m$ is odd, and $\alpha_{k}=e^{i \frac{(2 k+1) \pi}{m}}$ if $m$ is even.

Now we describe the so-called Dirichlet-to-Neumann operator $R\left(\alpha_{k} t\right): C^{\infty}(Y) \rightarrow$ $C^{\infty}(Y)$ associated to $\Delta_{M, D}+\alpha_{k} t$ on $Y$. Let $P_{i}\left(\alpha_{k} t\right): C^{\infty}(Y) \rightarrow C^{\infty}\left(M_{i}\right)$ be the Poisson operator on $Y$ associated to $\Delta_{M, D}+\alpha_{k} t$, which is characterized by the following equations (for details see [3], [4, [8]):

$$
B P_{i}\left(\alpha_{k} t\right)=I d_{Y}, \quad D P_{i}\left(\alpha_{k} t\right)=0, \quad\left(\Delta_{M}+\alpha_{k} t\right) P_{i}\left(\alpha_{k} t\right)=0 .
$$

Then $R\left(\alpha_{k} t\right)$ is defined by the composition of the following maps:

$$
\begin{gathered}
C^{\infty}(Y) \stackrel{\delta_{i a}}{\longrightarrow} C^{\infty}(Y) \oplus C^{\infty}(Y) \stackrel{\left(P_{1}\left(\alpha_{k} t\right), P_{2}\left(\alpha_{k} t\right)\right)}{\longrightarrow} C^{\infty}\left(M_{1}\right) \oplus C^{\infty}\left(M_{2}\right) \\
\stackrel{\left(C_{1}, C_{2}\right)}{\longrightarrow} C^{\infty}(Y) \oplus C^{\infty}(Y) \stackrel{\delta_{i f}}{\longrightarrow} C^{\infty}(Y),
\end{gathered}
$$

where $\delta_{i a}(g)=(g, g), C_{1}\left(\phi_{1}\right)=\left.\left(\partial_{u} \phi_{1}\right)\right|_{Y}, C_{2}\left(\phi_{2}\right)=\left.\left(\partial_{u} \phi_{2}\right)\right|_{Y}$ and $\delta_{i f}(g, h)=g-h$. It is known that $R\left(\alpha_{k} t\right)$ is a $\Psi D O$ of order 1 (cf. Theorem 2.1) and by choosing $\pi$ as an Agmon angle, $\log \operatorname{Det} R\left(\alpha_{k} t\right)$ is well defined. The following theorem is due to Burghelea, Friedlander and Kappeler ([8], see also [3] and [4]). 


\section{Theorem 1.1.}

$$
\begin{aligned}
\log \operatorname{Det}\left(\Delta_{M, D_{m}}^{m}+t^{m}\right) & -\log \operatorname{Det}\left(\Delta_{M_{1}, B_{m}, D_{m}}^{m}+t^{m}\right)-\log \operatorname{Det}\left(\Delta_{M_{2}, B_{m}, D_{m}}^{m}+t^{m}\right) \\
= & -\sum_{k=-\left[\frac{m}{2}\right]}^{\left[\frac{m-1}{2}\right]} c_{k}+\sum_{k=-\left[\frac{m}{2}\right]}^{\left[\frac{m-1}{2}\right]} \log \operatorname{Det} R\left(\alpha_{k} t\right),
\end{aligned}
$$

where $c_{k}$ is the zero coefficient in the asymptotic expansion of $\log \operatorname{Det} R\left(\alpha_{k} t\right)$ as $t \rightarrow \infty$.

Remark. In [3] and [8], Theorem 1.1 was proved only in the case $Z=\emptyset$. However, the proof can be extended without any modification to the case that $Z$ is non-empty.

The purpose of this paper is to compute the zero coefficients in Theorem 1.1 under the assumption of the product structures on $N$ and $\left.E\right|_{N}$, and then to apply this result to prove the adiabatic decomposition theorems for the zeta-determinant of a Laplacian and the analytic torsion. We first have the following theorem.

Theorem 1.2. We assume the product structures of $M$ and $E$ on $N$ and $\Delta_{M}=$ $-\partial_{u}^{2}+\Delta_{Y}$ on $N$. Then $\sum_{k} c_{k}=m \log 2 \cdot\left(\zeta_{\Delta_{Y}}(0)+\operatorname{dimKer} \Delta_{Y}\right)$.

Setting $t=0$, we get the following corollary.

Corollary 1.3. We further assume that $\Delta_{M, D}$ is invertible. Then

$$
\begin{aligned}
\log \operatorname{Det} \Delta_{M, D} & -\log \operatorname{Det} \Delta_{M_{1}, B, D}-\log \operatorname{Det} \Delta_{M_{2}, B, D} \\
& -\log 2 \cdot\left(\zeta_{\Delta_{Y}}(0)+\operatorname{dim} \operatorname{Ker} \Delta_{Y}\right)+\log \operatorname{Det} R .
\end{aligned}
$$

Remarks. (1) If $\operatorname{dim} Y$ is odd, it is well-known that $\zeta_{\Delta_{Y}}(0)+\operatorname{dimKer} \Delta_{Y}=0$. In this case, the assertion in Corollary 1.3 can be written as follows:

$$
\log \operatorname{Det} \Delta_{M, D}-\log \operatorname{Det} \Delta_{M_{1}, B, D}-\log \operatorname{Det} \Delta_{M_{2}, B, D}=\log \operatorname{Det} R,
$$

which was observed in [7].

(2) Theorem 1.1, Theorem 1.2 and Corollary 1.3 also hold when we impose the absolute (or the relative) boundary condition on $Z$ (see Theorem 5.2).

The main idea of proving Theorem 1.2 is to show that under the assumption of the product structure, $R\left(\alpha_{k} t\right)$ can be expressed as $2 \sqrt{\Delta_{Y}+\alpha_{k} t}+$ a smoothing operator (Theorem 2.1). We are going to show this fact in the next section by using an observation, due to I.M. Gelfand (probably unpublished), that the Dirichlet-toNeumann operator satisfies a Ricatti type equation ( $c f .(2.2))$.

Now we apply Corollary 1.3 to discuss the adiabatic decomposition of the zetadeterminant of a Laplacian into the zeta-determinants of Laplacians with the Dirichlet and Neumann boundary conditions. Recall that $N$ is a collar neighborhood of $Y$, which is diffeomorphic to $[-1,1] \times Y$. We denote by $M_{r}$ the compact manifold with boundary obtained by attaching $N_{r+1}=[-r-1, r+1] \times Y$ to $M-\left(-\frac{1}{2}, \frac{1}{2}\right) \times Y$ by identifying $\left[-1,-\frac{1}{2}\right] \times Y$ with $\left[-r-1,-r-\frac{1}{2}\right] \times Y$ and $\left[\frac{1}{2}, 1\right] \times Y$ with $\left[r+\frac{1}{2}, r+1\right] \times Y$. We also denote by $M_{1, r}, M_{2, r}$ the manifolds with boundary which are obtained by attaching $[-r, 0] \times Y,[0, r] \times Y$ to $M_{1}, M_{2}$ by identifying $Y$ with $\{-r\} \times Y$ and $Y$ with $\{r\} \times Y$, respectively. Then the bundle $E \rightarrow M$ and the Laplacian $\Delta_{M}$ on $M$ can be extended naturally to the bundle $E_{r} \rightarrow M_{r}$ and the Laplacian $\Delta_{M_{r}}$ on $M_{r}$.

To describe the next result, we need to define the operators $Q_{i}: C^{\infty}(Y) \rightarrow$ $C^{\infty}(Y)(i=1,2)$ by slightly modifying the Dirichlet-to-Neumann operator. For 
$f \in C^{\infty}(Y)$, choose $\phi_{i} \in C^{\infty}\left(M_{i}\right)$ satisfying $\Delta_{M_{i}} \phi_{i}=0,\left.\phi_{i}\right|_{Z}=0$ and $\left.\phi_{i}\right|_{Y}=f$. We define

$$
Q_{1}(f)=\left.\left(\partial_{u} \phi_{1}\right)\right|_{Y}, \quad Q_{2}(f)=\left.\left(-\partial_{u} \phi_{2}\right)\right|_{Y} .
$$

Then each $Q_{i}$ is an elliptic $\Psi$ DO of order 1 (cf. Theorem 2.1), and the Dirichletto-Neumann operator $R$ is $R=Q_{1}+Q_{2}$. The following is the second result of this paper.

Theorem 1.4. We assume that both $Q_{1}+\sqrt{\Delta_{Y}}$ and $Q_{2}+\sqrt{\Delta_{Y}}$ are invertible operators and $k=\operatorname{dimKer} \Delta_{Y}$. We further assume that $\Delta_{M_{r}, D}$ is invertible for $r$ large enough. Then

$$
\begin{array}{r}
\lim _{r \rightarrow \infty}\left\{\log \operatorname{Det}\left(\Delta_{M_{r}, D}\right)-\log \operatorname{Det}\left(\Delta_{M_{1, r}, B, D}\right)-\log \operatorname{Det}\left(\Delta_{M_{2, r}, B, D}\right)+k \log r\right\} \\
=\frac{1}{2} \log \operatorname{Det} \Delta_{Y} .
\end{array}
$$

Remarks. (1) If $\Delta_{Y}$ has non-trivial kernel, we define $\operatorname{Det} \Delta_{Y}$ from the zeta function $\zeta_{\Delta_{Y}}(s)$ consisting of only non-zero eigenvalues.

(2) If $\Delta_{M}$ is a connection Laplacian for a connection compatible with the inner product, each $Q_{i}$ is a non-negative operator (Lemma 4.3).

(3) Suppose that $\Delta_{M}=A^{2}$ for a Dirac operator $A$ which has the form $G\left(\partial_{u}+B\right)$ near $Y$ with $G$ a bundle automorphism satisfying

$$
G^{*}=-G, \quad G^{2}=-I d, \quad B^{*}=B, \quad G B=-B G .
$$

Here $G$ and $B$ do not depend on the normal coordinate $u$. Then the invertiblity of both $Q_{1}+\sqrt{B^{2}}$ and $Q_{2}+\sqrt{B^{2}}$ is equivalent to the non-existence of the extended $L^{2}$-solutions of $A_{M_{1, \infty}}, A_{M_{2, \infty}}$ on $M_{1, \infty}$ and $M_{2, \infty}$ (Corollary 4.5).

(4) Suppose that $\Delta_{M}$ is a connection Laplacian or a Dirac Laplacian for a connection compatible with the inner product, and $\Delta_{M, D}$ is invertible. Then the invertiblity of both $Q_{1}+\sqrt{\Delta_{Y}}$ and $Q_{2}+\sqrt{\Delta_{Y}}$ implies the invertiblity of $\Delta_{M_{r}, D}$ for $r$ large enough (Lemma 4.6).

Let $\tilde{M}_{1, r}$ be the double of $M_{1, r}$. Then it is a well-known fact that

$$
\log \operatorname{Det} \Delta_{\tilde{M}_{1, r}, D, D}=\log \operatorname{Det} \Delta_{M_{1, r}, C, D}+\log \operatorname{Det} \Delta_{M_{1, r}, B, D} .
$$

Combining this fact with Corollary 1.3 and Theorem 1.4, we have the following result.

Corollary 1.5. We assume the hypotheses in Theorem 1.4. Then:

(1) $\lim _{r \rightarrow \infty}\left\{\log \operatorname{Det}\left(\Delta_{M_{1, r}, C, D}\right)-\log \operatorname{Det}\left(\Delta_{M_{1, r}, B, D}\right)+k \log r\right\}=\frac{1}{2} \log \operatorname{Det}\left(\Delta_{Y}\right)$.

(2) $\lim _{r \rightarrow \infty}\left\{\log \operatorname{Det}\left(\Delta_{M_{r}, D}\right)-\log \operatorname{Det}\left(\Delta_{M_{1, r}, C, D}\right)-\log \operatorname{Det}\left(\Delta_{M_{2, r}, B, D}\right)\right\}=0$.

Finally we discuss the adiabatic decomposition of the analytic torsion into the analytic torsions with the absolute and relative boundary conditions.

Here we assume that $M$ is a closed manifold with a hypersurface $Y$ and $M$ has a product structure near $Y$. We define $M_{r}, M_{1, r}$ and $M_{2, r}$ as above so that $M_{r}=M_{1, r} \cup_{\{0\} \times Y} M_{2, r}$. Suppose that $\rho_{M_{r}}\left(\rho_{M_{1, r}}, \rho_{M_{2, r}}, \rho_{Y}\right)$ is an orthogonal representation of $\pi_{1}\left(M_{r}\right)\left(\pi_{1}\left(M_{1, r}\right), \pi_{1}\left(M_{2, r}\right), \pi_{1}(Y)\right)$ to $S O(n)$, respectively. Then we can define the analytic torsions $\tau\left(M_{r}, \rho_{M_{r}}\right), \tau_{a b s}\left(M_{i, r}, \rho_{M_{i, r}}\right), \tau_{r e l}\left(M_{i, r}, \rho_{M_{i, r}}\right)$ $(i=1,2), \tau\left(Y, \rho_{Y}\right)$ in the standard way (for the definitions, see Section 5 ). Our 
goal is to recover the Klimek-Wojciechowski result about the analytic torsion in [6] as follows.

First, let us consider $M_{1, r}$ (a manifold with boundary $Y$ ) only. For a given representation $\rho_{M_{1, r}}: \pi_{1}\left(M_{1, r}\right) \rightarrow S O(n)$ and the natural homomorphism $\iota_{Y}$ : $\pi_{1}(Y) \rightarrow \pi_{1}\left(M_{1, r}\right)$, define $\rho_{Y}: \pi_{1}(Y) \rightarrow S O(n)$ by $\rho_{Y}=\rho_{M_{1, r}} \circ \iota_{Y}$. We denote by $\Delta_{Y}^{q}\left(\Delta_{M_{1, r}}^{q}\right)$ the Hodge Laplacian acting on $q$-forms on $Y$ (on $M_{1, r}$ ) and valued in $E_{\rho_{Y}}\left(E_{\rho_{M_{1}, r}}\right)$, where $E_{\rho_{Y}}=\tilde{Y} \times_{\rho_{Y}} \mathbb{R}^{n}$ with $\tilde{Y}$ the universal covering space of $Y$ ( $E_{\rho_{M_{1}, r}}$ is defined in the same way). We define $Q_{1}^{q}$ the same way as in Theorem 1.4 with the bundle $E=\bigwedge^{q} T^{*} M_{1, r} \otimes E_{\rho_{M_{1, r}}}$. If necessary, by tensoring $\mathbb{C}$ on $E$, we regard $E$ as a complex vector bundle. Then we have the following theorem.

Theorem 1.6. Suppose that for each $q, Q_{1}^{q}+\left(\begin{array}{cc}\sqrt{\Delta_{Y}^{q}} & 0 \\ 0 & \sqrt{\Delta_{Y}^{q-1}}\end{array}\right)$ is an invertible operator on $\{-r\} \times Y$ and $H^{q}\left(M_{1, r} ; \rho_{M_{1, r}}\right), H^{q}\left(M_{1, r}, Y ; \rho_{M_{1, r}}\right)$ are trivial groups. Then

$$
\lim _{r \rightarrow \infty}\left\{\log \tau_{a b s}\left(M_{1, r}, \rho_{M_{1, r}}\right)-\log \tau_{r e l}\left(M_{1, r}, \rho_{M_{1, r}}\right)\right\}=\log \tau\left(Y ; \rho_{Y}\right) .
$$

Remark. If $Q_{1}^{q}+\left(\begin{array}{cc}\sqrt{\Delta_{Y}^{q}} & 0 \\ 0 & \sqrt{\Delta_{Y}^{q-1}}\end{array}\right)$ is invertible, by Corollary 4.5 there are no extended $L^{2}$-solutions of $d_{q}+d_{q}^{*}$ on $M_{1, \infty}$, which implies that $\operatorname{Ker} \Delta_{Y}^{q-1}=\operatorname{Ker} \Delta_{Y}^{q}=0(c f$. [1], [2], [5]).

Next, we consider the closed manifold $M_{r}$ and manifolds with boundary $M_{i, r}$ $(i=1,2)$. For a given representation $\rho_{M_{r}}: \pi_{1}\left(M_{r}\right) \rightarrow S O(n)$ and the natural homomorphisms $\iota_{M_{i, r}}: \pi_{1}\left(M_{i, r}\right) \rightarrow \pi_{1}\left(M_{r}\right), \iota_{Y}: \pi_{1}(Y) \rightarrow \pi_{1}\left(M_{i, r}\right)$, define $\rho_{M_{i, r}}:$ $\pi_{1}\left(M_{i, r}\right) \rightarrow S O(n), \rho_{Y}: \pi_{1}(Y) \rightarrow S O(n)$ by $\rho_{M_{i, r}}=\rho_{M_{r}} \circ \iota_{M_{i, r}}, \rho_{Y}=\rho_{M_{i, r}} \circ \iota_{Y}$. We also define $\Delta_{Y}^{q}, Q_{1}^{q}$ and $Q_{2}^{q}$ as in Theorem 1.6.

Theorem 1.7. Suppose that, for each $q$,

$$
Q_{1}^{q}+\left(\begin{array}{cc}
\sqrt{\Delta_{Y}^{q}} & 0 \\
0 & \sqrt{\Delta_{Y}^{q-1}}
\end{array}\right) \text { and } Q_{2}^{q}+\left(\begin{array}{cc}
\sqrt{\Delta_{Y}^{q}} & 0 \\
0 & \sqrt{\Delta_{Y}^{q-1}}
\end{array}\right)
$$

are invertible operators on $\{-r\} \times Y,\{r\} \times Y$, and $H^{q}\left(M_{r} ; \rho_{M_{r}}\right), H^{q}\left(M_{1, r} ; \rho_{M_{1, r}}\right)$, $H^{q}\left(M_{2, r}, Y ; \rho_{M_{2, r}}\right)$ are trivial groups. Then:

(1) $\lim _{r \rightarrow \infty}\left(\log \operatorname{Det} \Delta_{M_{r}}^{q}-\log \operatorname{Det} \Delta_{M_{1, r}, a b s}^{q}-\log \operatorname{Det} \Delta_{M_{2, r}, r e l}^{q}\right)=0$.

(2) $\lim _{r \rightarrow \infty}\left(\log \tau\left(M_{r} ; \rho_{M_{r}}\right)-\log \tau_{a b s}\left(M_{1, r} ; \rho_{M_{1, r}}\right)-\log \tau_{r e l}\left(M_{2, r} ; \rho_{M_{2, r}}\right)\right)=0$.

Remark. Recently J. Park and K. Wojciechowski proved the following result in [10. Suppose that $M$ is an odd-dimensional compact manifold with $M=M_{1} \cup_{Y}$ $M_{2}$ and $D$ is a Dirac operator acting on smooth sections of a Clifford module bundle $E$ with $D=G\left(\partial_{u}+B\right)$ near $Y$. Denote by $P_{>}, P_{<}$the Atiyah-PatodiSinger boundary conditions projecting the positive and negative eigenspaces of $B$, respectively. Assume that

$$
\operatorname{Ker} B=\{0\}, \quad \operatorname{Ker}_{L^{2}} D_{1, \infty}=\operatorname{Ker}_{L^{2}} D_{2, \infty}=\{0\},
$$

where $\operatorname{Ker}_{L^{2}} D_{i, \infty}$ is the set of all extended $L^{2}$-solutions of $D_{i, \infty}$ on $M_{i, \infty}$. Then

$$
\lim _{r \rightarrow \infty}\left\{\log \operatorname{Det} D_{r}^{2}-\log \operatorname{Det} D_{M_{1, r}, P_{>}}^{2}-\log \operatorname{Det} D_{M_{2, r}, P_{<}}^{2}\right\}=-\log 2 \cdot \zeta_{B^{2}}(0) .
$$


This result is the main motivation of this paper. In [9] we are going to recover this result by using the techniques in this paper.

\section{AsYmptotic SYMBOL OF $R\left(\alpha_{k} t\right)$}

In this section, we are going to describe the asymptotic symbol of $R\left(\alpha_{k} t\right)$. The following method was observed by I.M. Gelfand.

We start by defining $Q_{i}\left(\alpha_{k} t\right): C^{\infty}(Y) \rightarrow C^{\infty}(Y)(i=1,2)$ as follows. For $f \in C^{\infty}(Y)$, choose $\phi_{i} \in C^{\infty}\left(M_{i}\right)$ such that

$$
\left(\Delta_{M_{i}}+\alpha_{k} t\right) \phi_{i}=0,\left.\quad \phi_{i}\right|_{Y}=f,\left.\quad \phi_{i}\right|_{Z}=0 .
$$

Then we define

$$
Q_{1}\left(\alpha_{k} t\right)(f)=\left.\left(\partial_{u} \phi_{1}\right)\right|_{Y} \quad \text { and } \quad Q_{2}\left(\alpha_{k} t\right)(f)=\left.\left(-\partial_{u} \phi_{1}\right)\right|_{Y} .
$$

From this definition, we get

$$
R\left(\alpha_{k} t\right)=Q_{1}\left(\alpha_{k} t\right)+Q_{2}\left(\alpha_{k} t\right)
$$

and it's enough to consider $Q_{1}\left(\alpha_{k} t\right)$ only. From now on we denote $Q_{1}\left(\alpha_{k} t\right)$ simply by $Q\left(\alpha_{k} t\right)$.

For $f \in C^{\infty}(Y)$, let $\varphi$ be a solution of $\Delta_{M_{1}}+\alpha_{k} t$ with $\left.\varphi\right|_{Y}=f$ and $\left.\phi\right|_{Z}=0$. Then

$$
\frac{d}{d u} \varphi(u, y)=Q_{u}\left(\alpha_{k} t\right) \varphi(u, y)
$$

where $Q_{u}\left(\alpha_{k} t\right)$ is defined similarly to $Q\left(\alpha_{k} t\right)=Q_{0}\left(\alpha_{k} t\right)$ at the level $\{u\} \times Y$ :

$$
\frac{d^{2}}{d u^{2}} \varphi(u, y)=\left(\frac{d}{d u} Q_{u}\left(\alpha_{k} t\right)\right) \varphi(u, y)+Q_{u}\left(\alpha_{k} t\right)^{2} \varphi(u, y) .
$$

For $0 \leq u<1$,

$$
\left(\Delta_{Y}+\alpha_{k} t\right) \varphi(u, y)=\left(\frac{d}{d u} Q_{u}\left(\alpha_{k} t\right)\right) \varphi(u, y)+Q_{u}\left(\alpha_{k} t\right)^{2} \varphi(u, y) .
$$

Consequently, for $0 \leq u<1$,

$$
\frac{d}{d u} Q_{u}\left(\alpha_{k} t\right)=-Q_{u}\left(\alpha_{k} t\right)^{2}+\left(\Delta_{Y}+\alpha_{k} t\right) .
$$

Now let us consider the asymptotic symbol of $Q_{u}\left(\alpha_{k} t\right)$ as follows:

$$
\sigma\left(Q_{u}\left(\alpha_{k} t\right)\right) \sim q_{1}(u, y, \xi)+q_{0}(u, y, \xi)+\cdots+q_{1-j}(u, y, \xi)+\cdots,
$$

where $q_{1-j}(u, y, \xi)$ is the homogeneous part of $\sigma\left(Q_{u}\left(\alpha_{k} t\right)\right)$ of order $1-j$ with respect to $\xi$. Then

$$
\sigma\left(\frac{d}{d u} Q_{u}\left(\alpha_{k} t\right)\right) \sim \frac{d}{d u} q_{1}(u, y, \xi)+\frac{d}{d u} q_{0}(u, y, \xi)+\cdots+\frac{d}{d u} q_{1-j}(u, y, \xi)+\cdots
$$

Note that

$$
\begin{aligned}
\sigma\left(Q_{u}\left(\alpha_{k} t\right)^{2}\right) & \sim \sum_{k=0}^{\infty} \sum_{\substack{\omega \mid+i+j=k \\
i, j \geq 0}} \frac{1}{\omega !} d_{\xi}^{\omega} q_{1-i}(u, y, \xi) \cdot D_{y}^{\omega} q_{1-j}(u, y, \xi) \\
& =q_{1}^{2}(u, y, \xi)+\left(d_{\xi} q_{1} D_{y} q_{1}+q_{0} q_{1}+q_{1} q_{0}\right)+\cdots
\end{aligned}
$$

Suppose that

$$
\sigma\left(\Delta_{Y}+\alpha_{k} t\right)=\left(p_{2}(y, \xi)+\alpha_{k} t I d\right)+p_{1}(y, \xi)+p_{0}(y, \xi) .
$$


Since $\frac{d}{d u} Q_{u}\left(\alpha_{k} t\right)$ is a $\Psi$ DO of order $1, q_{1}^{2}(u, y, \xi)=p_{2}(y, \xi)+\alpha_{k} t I d$. Applying the argument of Lemma 3.3 in [8] to the double of a manifold with boundary, one can show that

$$
q_{1}(u, y, \xi)=\sqrt{p_{2}(y, \xi)+\alpha_{k} t I d} .
$$

Hence $q_{1}$ does not depend on $u$, and $\frac{d}{d u} q_{1}(u, y, \xi)=0$. Again, from (2.2), (2.3) and (2.4), since $q_{1}$ is a scalar matrix, $\left(d_{\xi} q_{1} D_{y} q_{1}+2 q_{1} q_{0}\right)=p_{1}(y, \xi)$ and

$$
q_{0}(u, y, \xi)=\left(2 q_{1}(y, \xi)\right)^{-1}\left(p_{1}(y, \xi)-d_{\xi} q_{1}(y, \xi) \cdot D_{y} q_{1}(y, \xi)\right) \text {. }
$$

Hence $q_{0}(u, y, \xi)$ does not depend on $u$, and $\frac{d}{d u} q_{0}(u, y, \xi)=0$. In general,

$$
q_{-1}=\left(2 q_{1}\right)^{-1}\left\{-\sum_{\substack{|\omega|+i+j=2 \\ 0 \leq i, j \leq 1}} \frac{1}{\omega !} d_{\xi}^{\omega} q_{1-i}(y, \xi) \cdot D_{y}^{\omega} q_{1-j}(y, \xi)+p_{0}(y, \xi)\right\}
$$

and for $k \geq 3$,

$$
q_{1-k}=\left(2 q_{1}\right)^{-1}\left\{-\sum_{\substack{|\omega|+i+j=k \\ 0 \leq i, j \leq k-1}} \frac{1}{\omega !} d_{\xi}^{\omega} q_{1-i}(y, \xi) \cdot D_{y}^{\omega} q_{1-j}(y, \xi)\right\} .
$$

Hence, each $q_{1-k}$ does not depend on $u$, and this implies that $\frac{d}{d u} Q_{u}\left(\alpha_{k} t\right)$ is a smoothing operator. Setting $u=0$ in (2.2), we see that

$$
Q\left(\alpha_{k} t\right)^{2}=\left(\Delta_{Y}+\alpha_{k} t\right)+\text { a smoothing operator, }
$$

and we get the following theorem.

Theorem 2.1. Under the assumption of the product structure near $N$, we have the following:

(1) $Q\left(\alpha_{k} t\right)=\sqrt{\Delta_{Y}+\alpha_{k} t}+$ a smoothing operator.

(2) $R\left(\alpha_{k} t\right)=2 \sqrt{\Delta_{Y}+\alpha_{k} t}+$ a smoothing operator.

Proof. It's enough to show the first statement. From (2.5) we have

$$
Q\left(\alpha_{k} t\right)=\sqrt{\Delta_{Y}+\alpha_{k} t}+A,
$$

where $A$ is an operator of order 0 . Squaring both sides and using (2.6), we have

$$
\begin{aligned}
Q\left(\alpha_{k} t\right)^{2} & =\left(\Delta_{Y}+\alpha_{k} t\right)+\sqrt{\Delta_{Y}+\alpha_{k} t} A+A \sqrt{\Delta_{Y}+\alpha_{k} t}+A^{2} \\
& =\left(\Delta_{Y}+\alpha_{k} t\right)+\text { a smoothing operator. }
\end{aligned}
$$

Hence $\sqrt{\Delta_{Y}+\alpha_{k} t} A+A \sqrt{\Delta_{Y}+\alpha_{k} t}+A^{2}$ is a smoothing operator, which implies that $A$ is a smoothing operator.

3. Computation of the zero Coefficient of $\log \operatorname{Det} R\left(\alpha_{k} t\right)$ as $t \rightarrow \infty$

It is shown in 3] that $\log \operatorname{Det} R\left(\alpha_{k} t\right)$ has an asymptotic expansion as $t \rightarrow \infty$ and each coefficient can be computed by the asymptotic symbol of $R\left(\alpha_{k} t\right)$. Hence, from Theorem 2.1, $\log \operatorname{Det} R\left(\alpha_{k} t\right)$ and $\log \operatorname{Det}\left(2 \sqrt{\Delta_{Y}+\alpha_{k} t}\right)$ have the same asymptotic expansions as $t \rightarrow \infty$. In this section, we are going to compute the asymptotic expansion of $\log \operatorname{Det}\left(2 \sqrt{\Delta_{Y}+\alpha_{k} t}\right)$ by using the method in [12].

Note that

$$
\log \operatorname{Det}\left(2 \sqrt{\Delta_{Y}+\alpha_{k} t}\right)=\log 2 \cdot \zeta_{\left(\Delta_{Y}+\alpha_{k} t\right)}(0)+\frac{1}{2} \log \operatorname{Det}\left(\Delta_{Y}+\alpha_{k} t\right),
$$


and we are going to consider $\log \operatorname{Det}\left(\Delta_{Y}+\alpha_{k} t\right)$. Since $\operatorname{Re}\left(\alpha_{k}\right)$ is possibly negative, we avoid this difficulty as follows. Put $\alpha_{k}=e^{i \theta_{k}}$ with $\theta_{k}=\frac{2 k \pi}{m}$ for $m$ odd and $\frac{(2 k+1) \pi}{m}$ for $m$ even. Choose an angle $\phi_{k}$ with $0 \leq\left|\phi_{k}\right|<\frac{\pi}{2}$ so that $\operatorname{Re}\left(e^{i\left(\theta_{k}-\phi_{k}\right)}\right)>$ 0. (In fact, if $0 \leq\left|\theta_{k}\right|<\frac{\pi}{2}$, we choose $\phi_{k}=0$.) Then

$$
\begin{array}{r}
\log \operatorname{Det}\left(\Delta_{Y}+\alpha_{k} t\right)=\log \operatorname{Det}\left\{e^{i \phi_{k}}\left(e^{-i \phi_{k}} \Delta_{Y}+e^{i\left(\theta_{k}-\phi_{k}\right)} t\right)\right\} \\
=-\left.\frac{d}{d s}\right|_{s=0}\left\{e^{-i \phi_{k} s} \zeta_{\left(e^{-i \phi_{k}} \Delta_{Y}+e^{i\left(\theta_{k}-\phi_{k}\right)} t\right)}(s)\right\} \\
=i \phi_{k} \zeta_{\left(e^{-i \phi_{k}} \Delta_{Y}+e^{i\left(\theta_{k}-\phi_{k}\right)} t\right)}(0)+\log \operatorname{Det}\left(e^{-i \phi_{k}} \Delta_{Y}+e^{i\left(\theta_{k}-\phi_{k}\right)} t\right) .
\end{array}
$$

Put $\tilde{\theta}_{k}=\theta_{k}-\phi_{k}$. Then

$$
\begin{aligned}
\zeta_{\left(e^{-i \phi_{k}} \Delta_{Y}+e^{i\left(\theta_{k}-\phi_{k}\right)} t\right)}(s) & =\frac{1}{\Gamma(s)} \int_{0}^{\infty} r^{s-1} \operatorname{Tr} e^{-r\left(e^{-i \phi_{k}} \Delta_{Y}+e^{i \tilde{\theta}_{k}} t\right)} d r \\
& =\frac{1}{\Gamma(s)} \int_{0}^{\infty} r^{s-1} e^{-r t e^{i \tilde{\theta}_{k}}} T r e^{-r e^{-i \phi_{k}} \Delta_{Y}} d r .
\end{aligned}
$$

The following lemma is a well-known fact.

Lemma 3.1. As $r \rightarrow 0$, we have the following asymptotic expansion:

$$
\text { Tre } e^{-r e^{-i \phi_{k}} \Delta_{Y}} \sim b_{1} r^{-\frac{m-1}{2}}+b_{2} r^{-\frac{m-2}{2}}+\cdots+b_{m}+b_{m-1} r^{\frac{1}{2}}+\cdots
$$

with $b_{m}=\zeta_{\Delta_{Y}}(0)+\operatorname{dim} \operatorname{Ker} \Delta_{Y}$.

Now we are going to compute the asymptotic expansion of $\zeta_{\left(e^{-i \phi_{k}} \Delta_{Y}+e^{\left.i \tilde{\theta}_{k} t\right)}\right.}(s)$ as $t \rightarrow \infty$ :

$$
\begin{aligned}
& \zeta_{\left(e^{-i \phi_{k}} \Delta_{Y}+e^{i \tilde{\theta}_{k}} t\right)}(s)=\frac{1}{\Gamma(s)} \int_{0}^{\infty} r^{s-1} e^{-r t e^{i \tilde{\theta}_{k}}} \operatorname{Tr} e^{-r e^{-i \phi_{k}} \Delta_{Y}} d r \\
& =\frac{1}{\Gamma(s)} \int_{0}^{\infty}\left(\frac{u}{t}\right)^{s-1} e^{-u e^{i \tilde{\theta}_{k}}} \operatorname{Tr} e^{-\frac{u}{t} e^{-i \phi_{k}} \Delta_{Y}} \frac{1}{t} d u \\
& =t^{-s} \frac{1}{\Gamma(s)} \int_{0}^{\infty} u^{s-1} e^{-u e^{i \tilde{\theta}_{k}}} \operatorname{Tr} e^{-\frac{u}{t} e^{-i \phi_{k}} \Delta_{Y}} d u \text {. }
\end{aligned}
$$

As $t \rightarrow \infty$,

$$
\begin{array}{r}
\zeta_{\left(e^{-i \phi_{k}} \Delta_{Y}+e^{\left.i \tilde{\theta}_{k} t\right)}\right.}(s) \\
\sim t^{-s} \sum_{j=1}^{\infty} \frac{1}{\Gamma(s)} b_{j} \int_{0}^{\infty} u^{s-1}\left(\frac{u}{t}\right)^{\frac{j-m}{2}} e^{-u e^{i \tilde{\theta}_{k}}} d u \\
=\sum_{j=1}^{\infty} b_{j} t^{-s+\frac{m-j}{2}} \frac{1}{\Gamma(s)} \int_{0}^{\infty} u^{s+\frac{j-m}{2}-1} e^{-u e^{i \tilde{\theta}_{k}}} d u \\
=\sum_{j=1}^{\infty} b_{j} t^{-s+\frac{m-j}{2}} \frac{1}{\Gamma(s)}\left(e^{-i \tilde{\theta}_{k}}\right)^{s+\frac{j-m}{2}} \int_{0}^{\infty}\left(u e^{i \tilde{\theta}_{k}}\right)^{s+\frac{j-m}{2}-1} e^{-u e^{i \tilde{\theta}_{k}}}\left(e^{i \tilde{\theta}_{k}}\right) d u .
\end{array}
$$

Consider the contour integral $\int_{C} z^{s+\frac{j-m}{2}-1} e^{-z} d z$ for $R e s>\frac{m-j}{2}$, where

$$
\begin{aligned}
C=\left\{r e^{i \tilde{\theta}_{k}} \mid \epsilon \leq r \leq R\right\} \cup\left\{\epsilon e^{i \theta} \mid 0 \leq \theta\right. & \left.\leq \tilde{\theta}_{k}\right\} \\
& \cup\{r \mid \epsilon \leq r \leq R\} \cup\left\{R e^{i \theta} \mid 0 \leq \theta \leq \tilde{\theta}_{k}\right\}
\end{aligned}
$$

and oriented counterclockwise. Then one can check that

$$
\int_{0}^{\infty}\left(u e^{i \tilde{\theta}_{k}}\right)^{s+\frac{j-m}{2}-1} e^{-u e^{i \tilde{\theta}_{k}}}\left(e^{i \tilde{\theta}_{k}}\right) d u=\int_{0}^{\infty} r^{s+\frac{j-m}{2}-1} e^{-r} d r=\Gamma\left(s+\frac{j-m}{2}\right) .
$$


We therefore obtain the following asymptotic expansion for $t \rightarrow \infty$ :

$$
\begin{gathered}
\zeta_{\left(e^{-i \phi_{k}} \Delta_{Y}+e^{\left.i \tilde{\theta}_{k} t\right)}\right.}(s) \sim \sum_{j=1}^{\infty} b_{j}\left(e^{-i \tilde{\theta}_{k}}\right)^{s+\frac{j-m}{2}} \frac{\Gamma\left(s+\frac{j-m}{2}\right)}{\Gamma(s)} t^{-s+\frac{m-j}{2}} \\
=s \sum_{\substack{j=1 \\
j \neq m}}^{\infty} b_{j}\left(e^{-i \tilde{\theta}_{k}}\right)^{s+\frac{j-m}{2}} \frac{\Gamma\left(s+\frac{j-m}{2}\right)}{\Gamma(s+1)} t^{-s+\frac{m-j}{2}}+b_{m} e^{-i \tilde{\theta}_{k} s} t^{-s} .
\end{gathered}
$$

This gives the asymptotic expansion of $\zeta_{\left(e^{-i \phi_{k}} \Delta_{Y}+e^{\left.i \tilde{\theta}_{k} t\right)}\right.}(s)$ as $t \rightarrow \infty$. In view of Theorem 1.1 we are mainly interested in the zero coefficients in the asymptotic expansions of $\zeta_{\left(e^{-i \phi_{k}} \Delta_{Y}+e^{i \tilde{\theta}_{k}} t\right)}(0)$ and $\zeta_{\left(e^{-i \phi_{k}} \Delta_{Y}+e^{\left.i \tilde{\theta}_{k} t\right)}\right.}^{\prime}(0)$ as $t \rightarrow \infty$.

First, setting $s=0$, the zero coefficient $\pi_{0}\left(\zeta_{\left(e^{-i \phi_{k}} \Delta_{Y}+e^{i \tilde{\theta}_{k}} t\right)}(0)\right)$ in the asymptotic expansion of $\zeta_{\left(e^{-i \phi_{k}} \Delta_{Y}+e^{\left.i \tilde{\theta}_{k} t\right)}\right.}(0)$ is the following:

$$
\pi_{0}\left(\zeta_{\left(e^{-i \phi_{k}} \Delta_{Y}+e^{\left.i \tilde{\theta}_{k} t\right)}\right.}(0)\right)=b_{m}=\zeta_{\Delta_{Y}}(0)+\operatorname{dimKer} \Delta_{Y} .
$$

Taking the derivative at $s=0$, the zero coefficient of $\zeta_{\left(e^{-i \phi_{k}} \Delta_{Y}+e^{\left.i \tilde{\theta}_{k} t\right)}\right.}^{(0)}$ can be obtained only in the term $b_{m} e^{-i \tilde{\theta}_{k} s} t^{-s}$. Hence, by (3.2) and (3.3), the zero coefficient $\pi_{0}\left(\Delta_{Y}+\alpha_{k} t\right)$ in the asymptotic expansion of $\log \operatorname{Det}\left(\Delta_{Y}+\alpha_{k} t\right)$ as $t \rightarrow \infty$ is

$$
\begin{aligned}
\pi_{0}\left(\Delta_{Y}+\alpha_{k} t\right) & =i \phi_{k}\left(\zeta_{\Delta_{Y}}(0)+\operatorname{dimKer} \Delta_{Y}\right)+i\left(\theta_{k}-\phi_{k}\right)\left(\zeta_{\Delta_{Y}}(0)+\operatorname{dimKer} \Delta_{Y}\right) \\
& =i \theta_{k}\left(\zeta_{\Delta_{Y}}(0)+\operatorname{dimKer} \Delta_{Y}\right) .
\end{aligned}
$$

We summarize the above computations as follows.

Proposition 3.2. The zero coefficients in the asymptotic expansions of

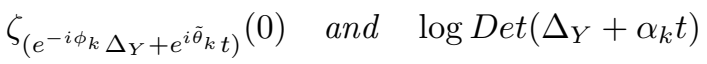

as $t \rightarrow \infty$ are the following:

(1) $\pi_{0}\left(\zeta_{\left(e^{-i \phi_{k}} \Delta_{Y}+e^{\left.i \tilde{\theta}_{k} t\right)}\right.}(0)\right)=\zeta_{\Delta_{Y}}(0)+\operatorname{dimKer} \Delta_{Y}$.

(2) $\pi_{0}\left(\Delta_{Y}+\alpha_{k} t\right)=i \theta_{k}\left(\zeta_{\Delta_{Y}}(0)+\operatorname{dimKer} \Delta_{Y}\right)$, where $\alpha_{k}=e^{i \theta_{k}}$.

Now we are ready to compute $c=\sum_{k} c_{k}$ in Theorem 1.1. Since $\zeta_{\left(\Delta_{Y}+\alpha_{k} t\right)}(0)=$ $\zeta_{\left(e^{-i \phi_{k}} \Delta_{Y}+e^{\left.i \tilde{\theta}_{k} t\right)}\right.}(0)$, from (3.1) and Proposition 3.2 we get

$$
c_{k}=\log 2 \cdot\left(\zeta_{\Delta_{Y}}(0)+\operatorname{dimKer} \Delta_{Y}\right)+\frac{1}{2} i \theta_{k}\left(\zeta_{\Delta_{Y}}(0)+\operatorname{dimKer} \Delta_{Y}\right),
$$

and hence

$$
\sum_{k} c_{k}=m \log 2 \cdot\left(\zeta_{\Delta_{Y}}(0)+\operatorname{dim} \operatorname{Ker} \Delta_{Y}\right) .
$$

This completes the proof of Theorem 1.2.

\section{The Adiabatic DECOMPOSITION OF THE ZETA-DETERMINANT OF A LAPLACIAN}

In this section we are going to prove Theorem 1.4. Recall that

$$
M_{1, r}=M_{1} \cup_{Y}[-r, 0] \times Y, \quad M_{2, r}=M_{2} \cup_{Y}[0, r] \times Y,
$$

where we identify $Y$ with $\{-r\} \times Y$ and $Y$ with $\{r\} \times Y$. Then

$$
M_{r}=M_{1, r} \cup_{\{0\} \times Y} M_{2, r} .
$$

Throughout this section we denote $\{r\} \times Y$ by $Y_{r}$, the Dirichlet (Neumann) condition on $Y_{r}$ by $B_{r}\left(C_{r}\right)$ and the Dirichlet condition on $Z$ by $D$. We assume that $\Delta_{M, D}$ 
is invertible. Then, under certain conditions, $\Delta_{M_{r}, D}$ is also invertible for $r$ large enough (Lemma 4.6).

From the decomposition (4.1) and Corollary 1.3, we have

$$
\begin{aligned}
\log \operatorname{Det} \Delta_{M_{r}, D}=\log \operatorname{Det} \Delta_{M_{1, r}, B_{0}, D}+\log \operatorname{Det} \Delta_{M_{2, r}, B_{0}, D} \\
\\
-\log 2 \cdot\left(\zeta_{\Delta_{Y}}(0)+\operatorname{dim} \operatorname{Ker} \Delta_{Y}\right)+\log \operatorname{Det} R_{M_{r}} .
\end{aligned}
$$

From the decomposition $M_{r}=\left(M_{1} \cup M_{2}\right) \cup N_{r}$ with $N_{r}=[-r, r] \times Y$, we have

$$
\begin{aligned}
\log \operatorname{Det}_{M_{r}, D}= & \log \operatorname{Det} \Delta_{\left(M_{1} \cup M_{2}\right), B_{-r}, B_{r}, D}+\log \operatorname{Det} \Delta_{N_{r}, B_{-r}, B_{r}} \\
& -\log 2 \cdot\left(\zeta_{\Delta_{Y} \cup Y}(0)+\operatorname{dim} \operatorname{Ker} \Delta_{Y \cup Y}\right)+\log \operatorname{Det} R_{-r, r} \\
= & \log \operatorname{Det} \Delta_{M_{1}, B, D}+\log \operatorname{Det} \Delta_{M_{2}, B, D}+\log \operatorname{Det} \Delta_{N_{r}, B_{-r}, B_{r}} \\
& -2 \log 2 \cdot\left(\zeta_{\Delta_{Y}}(0)+\operatorname{dim} \operatorname{der} \Delta_{Y}\right)+\log \operatorname{Det} R_{-r, r},
\end{aligned}
$$

where $R_{-r, r}: C^{\infty}\left(Y_{-r}\right) \oplus C^{\infty}\left(Y_{r}\right) \rightarrow C^{\infty}\left(Y_{-r}\right) \oplus C^{\infty}\left(Y_{r}\right)$ is the Dirichlet-toNeumann operator corresponding to the decomposition $\left(M_{1} \cup M_{2}\right) \cup N_{r}$.

Put $N_{-r, 0}=[-r, 0] \times Y$ and $N_{0, r}=[0, r] \times Y$. Since $M_{1, r}=M_{1} \cup N_{-r, 0}$ and $M_{2, r}=M_{2} \cup N_{0, r}$, we have

$$
\begin{aligned}
& \log \operatorname{Det} \Delta_{M_{1, r}, B_{0}, D}= \log \operatorname{Det}_{M_{1}, B, D}+\log \operatorname{Det} \Delta_{N_{-r, 0}, B_{-r}, B_{0}} \\
&-\log 2 \cdot\left(\zeta_{\Delta_{Y}}(0)+\operatorname{dim} \operatorname{Ker} \Delta_{Y}\right)+\log \operatorname{Det} R_{M_{1, r}}, \\
& \log \operatorname{Det} \Delta_{M_{2, r}, B_{0}, D}=\log \operatorname{Det} \Delta_{M_{2}, B, D}+\log \operatorname{Det} \Delta_{N_{0, r}, B_{0}, B_{r}} \\
&-\log 2 \cdot\left(\zeta_{\Delta_{Y}}(0)+\operatorname{dimKer} \Delta_{Y}\right)+\log \operatorname{Det} R_{M_{2, r}} .
\end{aligned}
$$

Here $\Delta_{N_{-r, 0}, B_{-r}, B_{0}}=-\partial_{u}^{2}+\Delta_{Y}$ with the domain $\left\{\phi \in C^{\infty}\left(N_{-r, 0}\right)|\phi|_{Y_{-r}}=\left.\phi\right|_{Y_{0}}=\right.$ $0\}$ and $R_{M_{1, r}}$ is the Dirichlet-to-Neumann operator corresponding to the decomposition $M_{1, r}=M_{1} \cup([-r, 0] \times Y) . \Delta_{N_{0, r}, B_{0}, B_{r}}$ and $R_{M_{2, r}}$ are defined similarly.

Then from (4.2)-(4.5), we have

$$
\begin{gathered}
\quad-\log 2 \cdot\left(\zeta_{\Delta_{Y}}(0)+\operatorname{dimKer} \Delta_{Y}\right)+\log \operatorname{Det} R_{M_{r}} \\
=\log \operatorname{Det} \Delta_{N_{r}, B_{-r}, B_{r}}-\log \operatorname{Det} \Delta_{N_{-r, 0}, B_{-r}, B_{0}}-\log \operatorname{Det} \Delta_{N_{0, r}, B_{0}, B_{r}} \\
+\log \operatorname{Det} R_{-r, r}-\log \operatorname{Det} R_{M_{1, r}}-\log \operatorname{Det} R_{M_{2, r} .}
\end{gathered}
$$

From the decomposition of $N_{r}$ as

$$
N_{r}=([-r, 0] \times Y) \cup([0, r] \times Y),
$$

we have

$$
\begin{aligned}
\log \operatorname{Det} \Delta_{N_{r}, B_{-r}, B_{r}}-\log \operatorname{Det} \Delta_{N_{-r, 0}, B_{-r}, B_{0}}-\log \operatorname{Det} \Delta_{N_{0, r}, B_{0}, B_{r}} & \\
& =-\log 2 \cdot\left(\zeta_{\Delta_{Y}}(0)+\operatorname{dimKer} \Delta_{Y}\right)+\log \operatorname{Det} R_{N_{r}}
\end{aligned}
$$

where $R_{N_{r}}: C^{\infty}\left(Y_{0}\right) \rightarrow C^{\infty}\left(Y_{0}\right)$ is defined as follows. For $f \in C^{\infty}\left(Y_{0}\right)$, choose $\phi(u, y)$ so that $\left(-\partial_{u}^{2}+\Delta_{Y}\right) \phi=0$ on $N_{r}-Y_{0},\left.\phi\right|_{Y_{0}}=f,\left.\phi\right|_{Y_{-r}}=\left.\phi\right|_{Y_{r}}=0$. Then, $R_{N_{r}}(f)=\left.\left(\partial_{u}\left(\left.\phi\right|_{N_{-r, 0}}\right)-\partial_{u}\left(\left.\phi\right|_{N_{0, r}}\right)\right)\right|_{Y_{0}}$. Hence, we obtain from (4.6) and (4.7)

$$
\log \operatorname{Det}_{M_{r}}=\log \operatorname{Det}_{N_{r}}+\log \operatorname{Det} R_{-r, r}-\log \operatorname{Det} R_{M_{1, r}}-\log \operatorname{Det} R_{M_{2, r}} \text {. }
$$


Now we are going to find the spectrum of $R_{N_{r}}: C^{\infty}\left(Y_{0}\right) \rightarrow C^{\infty}\left(Y_{0}\right)$. For $f_{k} \in C^{\infty}\left(Y_{0}\right)$ with $\Delta_{Y} f_{k}=\lambda_{k} f_{k}$, we have

$$
\phi(u, y)=\left\{\begin{array}{r}
\left(e^{\sqrt{\lambda_{k}} u}+\frac{e^{-\sqrt{\lambda_{k}} r}}{e^{\sqrt{\lambda_{k}} r}-e^{-\sqrt{\lambda_{k}} r}}\left(e^{\sqrt{\lambda_{k}} u}-e^{-\sqrt{\lambda_{k}} u}\right)\right) f_{k}(y) \\
\text { for }(u, y) \in N_{-r, 0}, \\
\left(e^{-\sqrt{\lambda_{k}} u}-\frac{e^{-\sqrt{\lambda_{k}} r}}{e^{\sqrt{\lambda_{k}} r}-e^{-\sqrt{\lambda_{k}} r}}\left(e^{\sqrt{\lambda_{k}} u}-e^{-\sqrt{\lambda_{k}} u}\right)\right) f_{k}(y) \\
\text { for }(u, y) \in N_{0, r} .
\end{array}\right.
$$

Hence,

$$
R_{N_{r}}\left(f_{k}\right)=\left(2 \sqrt{\lambda_{k}}+\frac{4 \sqrt{\lambda_{k}} e^{-\sqrt{\lambda_{k}} r}}{e^{\sqrt{\lambda_{k}} r}-e^{-\sqrt{\lambda_{k}} r}}\right) f_{k},
$$

where we interpret $\frac{4 \sqrt{\lambda_{k}} e^{-\sqrt{\lambda_{k}} r}}{e^{\sqrt{\lambda_{k}} r}-e^{-\sqrt{\lambda_{k}} r}}$ as $\frac{2}{r}$ when $\lambda_{k}=0$. The spectrum of $R_{N_{r}}$ is

$$
\left\{2 \sqrt{\lambda_{k}}+\frac{4 \sqrt{\lambda_{k}} e^{-\sqrt{\lambda_{k}} r}}{e^{\sqrt{\lambda_{k}} r}-e^{-\sqrt{\lambda_{k}} r}} \mid \lambda_{k} \in \operatorname{Spec}\left(\Delta_{Y}\right)\right\} .
$$

Let $P_{K e r \Delta_{Y}}: C^{\infty}(Y) \rightarrow C^{\infty}(Y)$ be the orthogonal projection onto $\operatorname{Ker} \Delta_{Y}$. Then

$$
\begin{aligned}
\zeta_{R_{N_{r}}}(s)-\zeta_{\left(2 \sqrt{\Delta_{Y}}+\frac{2}{r} P_{K e r \Delta_{Y}}\right)}(s) & \\
= & \sum_{\lambda_{k} \neq 0}\left\{\left(2 \sqrt{\lambda_{k}}+\frac{4 \sqrt{\lambda_{k}} e^{-\sqrt{\lambda_{k}} r}}{e^{\sqrt{\lambda_{k}} r}-e^{-\sqrt{\lambda_{k}} r}}\right)^{-s}-\left(2 \sqrt{\lambda_{k}}\right)^{-s}\right\} .
\end{aligned}
$$

The following lemma can be checked easily.

Lemma 4.1. Let $A$ be an invertible elliptic operator of order $>0$, and $K_{r}$ a oneparameter family of trace class operators such that $\lim _{r \rightarrow \infty} \operatorname{Tr}\left(K_{r}\right)=0$. Then

$$
\lim _{r \rightarrow \infty} \log \operatorname{Det}\left(A+K_{r}\right)=\log \operatorname{Det} A .
$$

Proof. Note that

$$
\begin{aligned}
\log \operatorname{Det}\left(A+K_{r}\right)-\log \operatorname{Det} A & =\int_{0}^{1} \frac{d}{d t} \log \operatorname{Det}\left(A+t K_{r}\right) d t \\
& =\int_{0}^{1} \operatorname{Tr}\left(\left(A+t K_{r}\right)^{-1} K_{r}\right) d t
\end{aligned}
$$

If we denote by $\lambda_{0}$ the smallest eigenvalue of $|A|$, for $r$ large enough we have

$$
\left|\log \operatorname{Det}\left(A+K_{r}\right)-\log \operatorname{Det} A\right| \leq \frac{1}{2 \lambda_{0}} \operatorname{Tr}\left(K_{r}\right)
$$

and hence the result follows.

Applying Lemma 4.1 with $A=2 \sqrt{\Delta_{Y}}$ and $K_{r}=g_{r}\left(\Delta_{Y}\right)$ with

$$
g_{r}(x)=\frac{4 \sqrt{x} e^{-\sqrt{x} r}}{e^{\sqrt{x} r}-e^{-\sqrt{x} r}}
$$

on the orthogonal complement of $\operatorname{Ker} \Delta_{Y}$, we get the following equation:

$$
\lim _{r \rightarrow \infty}\left\{\log \operatorname{Det} R_{N_{r}}-\log \operatorname{Det}\left(2 \sqrt{\Delta_{Y}}+\frac{2}{r} P_{K e r \Delta_{Y}}\right)\right\}=0
$$


Since

$$
\begin{array}{r}
\log \operatorname{Det}\left(2 \sqrt{\Delta_{Y}}+\frac{2}{r} P_{K e r \Delta_{Y}}\right)=\log 2 \cdot\left(\zeta_{\Delta_{Y}}(0)+\operatorname{dimKer} \Delta_{Y}\right)+\frac{1}{2} \log \operatorname{Det} \Delta_{Y} \\
-\left(\operatorname{dimKer} \Delta_{Y}\right) \log r
\end{array}
$$

we get the following corollary.

\section{Corollary 4.2.}

$$
\begin{aligned}
\lim _{r \rightarrow \infty} & \left(\log \operatorname{Det} R_{N_{r}}+\left(\operatorname{dimKer} \Delta_{Y}\right) \log r\right) \\
\quad & \log 2 \cdot\left(\zeta_{\Delta_{Y}}(0)+\operatorname{dimKer} \Delta_{Y}\right)+\frac{1}{2} \log \operatorname{Det} \Delta_{Y} .
\end{aligned}
$$

Now we discuss the operators $R_{M_{1, r}}, R_{M_{2, r}}$ and $R_{-r, r}$. First, we can describe $R_{M_{1, r}}: C^{\infty}\left(Y_{-r}\right) \rightarrow C^{\infty}\left(Y_{-r}\right)$ as follows. For $f_{k} \in C^{\infty}\left(Y_{-r}\right)$ with $\Delta_{Y} f_{k}=\lambda_{k} f_{k}$, we choose the section $\phi \in C^{0}\left(M_{1, r}\right)$ satisfying $\Delta_{M_{1, r}} \phi=0$ on $M_{1, r}-Y_{-r},\left.\phi\right|_{Y_{-r}}=f_{k}$ and $\left.\phi\right|_{Z}=\left.\phi\right|_{Y_{0}}=0$. Then one can check that

$$
\begin{aligned}
R_{M_{1, r}}\left(f_{k}\right) & =Q_{1}\left(f_{k}\right)-\left.\left(\partial_{u}\left(\left.\phi\right|_{N_{-r, 0}}\right)\right)\right|_{Y_{-r}} \\
& =Q_{1}\left(f_{k}\right)+\left(\sqrt{\lambda_{k}}+\frac{2 \sqrt{\lambda_{k}} e^{-\sqrt{\lambda_{k}} r}}{e^{\sqrt{\lambda_{k}} r}-e^{-\sqrt{\lambda_{k}} r}}\right) f_{k} .
\end{aligned}
$$

In the same way,

$$
\begin{aligned}
R_{M_{2, r}}\left(f_{k}\right) & =Q_{2}\left(f_{k}\right)+\left.\left(\partial_{u}\left(\left.\phi\right|_{N_{0, r}}\right)\right)\right|_{Y_{r}} \\
& =Q_{2}\left(f_{k}\right)+\left(\sqrt{\lambda_{k}}+\frac{2 \sqrt{\lambda_{k}} e^{-\sqrt{\lambda_{k}} r}}{e^{\sqrt{\lambda_{k}} r}-e^{-\sqrt{\lambda_{k}} r}}\right) f_{k} .
\end{aligned}
$$

Similarly, $R_{-r, r}: C^{\infty}\left(Y_{-r}\right) \oplus C^{\infty}\left(Y_{r}\right) \rightarrow C^{\infty}\left(Y_{-r}\right) \oplus C^{\infty}\left(Y_{r}\right)$ is described as follows:

$$
\begin{aligned}
& R_{-r, r}\left(f_{k}, 0\right) \\
& \quad=\left(Q_{1}\left(f_{k}\right)+\left(\sqrt{\lambda_{k}}+\frac{2 \sqrt{\lambda_{k}} e^{-2 \sqrt{\lambda_{k}} r}}{e^{2 \sqrt{\lambda_{k}} r}-e^{-2 \sqrt{\lambda_{k}} r}}\right) f_{k},-\frac{2 \sqrt{\lambda_{k}}}{e^{2 \sqrt{\lambda_{k}} r}-e^{-2 \sqrt{\lambda_{k}} r}} f_{k}\right), \\
& R_{-r, r}\left(0, f_{k}\right) \\
& \quad=\left(-\frac{2 \sqrt{\lambda_{k}}}{e^{2 \sqrt{\lambda_{k}} r}-e^{-2 \sqrt{\lambda_{k}} r}} f_{k}, Q_{2}\left(f_{k}\right)+\left(\sqrt{\lambda_{k}}+\frac{2 \sqrt{\lambda_{k}} e^{-2 \sqrt{\lambda_{k}} r}}{e^{2 \sqrt{\lambda_{k}} r}-e^{-2 \sqrt{\lambda_{k}} r}}\right) f_{k}\right) .
\end{aligned}
$$

We therefore have

$$
R_{-r, r}=\left(\begin{array}{cc}
Q_{1}+\sqrt{\Delta_{Y}} & 0 \\
0 & Q_{2}+\sqrt{\Delta_{Y}}
\end{array}\right)+h_{r}\left(\Delta_{Y}\right)\left(\begin{array}{cc}
e^{-2 r \sqrt{\Delta_{Y}}} & -1 \\
-1 & e^{-2 r \sqrt{\Delta_{Y}}}
\end{array}\right),
$$

where $h_{r}(x)=\frac{2 \sqrt{x}}{e^{2 r \sqrt{x}}-e^{-2 r \sqrt{x}}}$ and $h_{r}\left(\Delta_{Y}\right)$ acts on $\operatorname{Ker} \Delta_{Y}$ as multiplication by $\frac{1}{2 r}$.

We are going to discuss the operators $Q_{i}$ and $Q_{i}+\sqrt{\Delta_{Y}}$. The following lemma can be checked by using integration by parts ( $c f$. Proposition 4.3 in [2]).

Lemma 4.3. Suppose that $\nabla$ is a connection which is compatible to the inner product on $M$. i.e. for any sections $s_{1}, s_{2} \in C^{\infty}(E)$ and a tangent vector $w$, $w\left(s_{1}, s_{2}\right)=\left(\nabla_{w} s_{1}, s_{2}\right)+\left(s_{1}, \nabla_{w} s_{2}\right)$. If $\Delta_{M}=\nabla^{*} \nabla$, then each $Q_{i}$ is a non-negative, self-adjoint operator. 
Next, let us consider a Dirac Laplacian for a Dirac operator $A$ which has the form $G\left(\partial_{u}+B\right)$ near the boundary $Y$, where $G$ is a bundle automorphism satisfying the conditions (1.1), and both $G$ and $B$ do not depend on the normal coordinate $u$. We refer to [5] for the following lemma ( $c f$. Lemma 3.1 in [5]).

Lemma 4.4. Let $\phi$ and $\psi$ be smooth sections on $M_{j}(j=1,2)$. Then

$$
\left\langle A_{M_{j}} \phi, \psi\right\rangle_{M_{j}}-\left\langle\phi, A_{M_{j}} \psi\right\rangle_{M_{j}}=\epsilon_{j}\left\langle\left.\phi\right|_{Y}, G\left(\left.\psi\right|_{Y}\right)\right\rangle_{Y},
$$

where $\epsilon_{j}=1$ for $j=2$ and $\epsilon_{j}=-1$ for $j=1$.

Suppose that for $f \in C^{\infty}(Y), \phi_{j}$ is the solution of $A_{M_{j}}^{2}$ with $\left.\phi_{j}\right|_{Y}=f,\left.\phi_{j}\right|_{Z}=0$. Then by Lemma 4.4

$$
\begin{aligned}
& \left\langle\left(Q_{1}+|B|\right) f, f\right\rangle_{Y}=\left\langle A_{M_{1}} \phi_{1}, A_{M_{1}} \phi_{1}\right\rangle_{M_{1}}+\langle(|B|-B) f, f\rangle_{Y}, \\
& \left\langle\left(Q_{2}+|B|\right) f, f\right\rangle_{Y}=\left\langle A_{M_{2}} \phi_{2}, A_{M_{2}} \phi_{2}\right\rangle_{M_{2}}+\langle(|B|+B) f, f\rangle_{Y} .
\end{aligned}
$$

As a consequence, $f \in \operatorname{Ker}\left(Q_{1}+|B|\right)$ if and only if $A_{M_{1}} \phi_{1}=0$ and $f \in \operatorname{ImP} P_{\geq}$; and hence on the cylinder part we can express $\phi_{1}$ as

$$
\phi_{1}=\sum_{j=1}^{k} a_{j} g_{j}+\sum_{\lambda_{j}>0} b_{j} e^{-\lambda_{j} u} h_{j},
$$

where $B g_{j}=0, B h_{j}=\lambda_{j} h_{j}$. This implies that $\phi_{1}$ is the restriction of an extended $L^{2}$-solution of $A_{M_{1}, \infty}$ on $M_{1, \infty}:=M_{1} \cup_{Y} Y \times[0, \infty)$. We can make a similar assertion for $\phi_{2}$ and have the following corollary (cf. Theorem 2.2 in [5], see also [1], [2]).

Corollary 4.5. The invertibility of $Q_{1}+\sqrt{B^{2}}$ and $Q_{2}+\sqrt{B^{2}}$ is equivalent to the non-existence of the extended $L^{2}$-solutions of $A_{M_{1, \infty}}$ and $A_{M_{2, \infty}}$ on $M_{1, \infty}$ and $M_{2, \infty}$. In particular, this condition implies that $\operatorname{Ker} B=0$.

Lemma 4.6. Suppose that $\Delta_{M}$ is either a connection Laplacian or a Dirac Laplacian for a connection compatible to the inner product as above, and $\Delta_{M, D}$ is invertible. If both $Q_{1}+\sqrt{\Delta_{Y}}$ and $Q_{2}+\sqrt{\Delta_{Y}}$ are invertible, then $R_{-r, r}$ and $\Delta_{M_{r, D}}$ are invertible for $r$ large enough.

Proof. We are going to show first that $R_{-r, r}$ is injective. Then this implies that $\Delta_{M_{r, D}}$ is injective. Since $\Delta_{M_{r, D}}$ is self-adjoint, $\Delta_{M_{r, D}}$ is invertible, and this implies again that $R_{-r, r}$ is also invertible ([3], [8]).

Putting $A_{r}=h_{r}\left(\Delta_{Y}\right)$ with $h_{r}(x)=\frac{2 \sqrt{x}}{e^{2 r \sqrt{x}}-e^{-2 r \sqrt{x}}}$, we get

$$
\begin{gathered}
\left\langle R_{-r, r}\left(\begin{array}{l}
f \\
g
\end{array}\right),\left(\begin{array}{l}
f \\
g
\end{array}\right)\right\rangle_{L^{2}(Y)} \\
=\left\langle\left(Q_{1}+\sqrt{\Delta_{Y}}\right) f, f\right\rangle+\left\langle\left(Q_{2}+\sqrt{\Delta_{Y}}\right) g, g\right\rangle \\
+\left\langle A_{r} e^{-2 r \sqrt{\Delta_{Y}}} f, f\right\rangle+\left\langle A_{r} e^{-2 r \sqrt{\Delta_{Y}}} g, g\right\rangle-\left\langle A_{r} g, f\right\rangle-\left\langle A_{r} f, g\right\rangle .
\end{gathered}
$$

Note that each $Q_{i}+\sqrt{\Delta_{Y}}$ is a non-negative operator by Lemma 4.3 and (4.10), (4.11). Let $\lambda_{0}$ be the minimum of the eigenvalues of $Q_{1}+\sqrt{\Delta_{Y}}$ and $Q_{2}+\sqrt{\Delta_{Y}}$. Since $\lim _{r \rightarrow \infty}\left\|A_{r}\right\|_{L^{2}}=0$, one can choose $r_{0}$ so that $\left\|A_{r}\right\|_{L^{2}}<\lambda_{0}$ for $r \geq r_{0}$. Then $R_{-r, r}$ is injective for $r \geq r_{0}$ and this completes the proof. 
In case both $Q_{1}+\sqrt{\Delta_{Y}}$ and $Q_{2}+\sqrt{\Delta_{Y}}$ are invertible, we can apply Lemma 4.1 directly.

Corollary 4.7. Assume that both $Q_{1}+\sqrt{\Delta_{Y}}$ and $Q_{2}+\sqrt{\Delta_{Y}}$ are invertible. Then:

(1) $\lim _{r \rightarrow \infty} \log \operatorname{Det} R_{M_{1, r}}=\log \operatorname{Det}\left(Q_{1}+\sqrt{\Delta_{Y}}\right)$.

(2) $\lim _{r \rightarrow \infty} \log \operatorname{Det} R_{M_{2, r}}=\log \operatorname{Det}\left(Q_{2}+\sqrt{\Delta_{Y}}\right)$.

(3) $\lim _{r \rightarrow \infty} \log \operatorname{Det} R_{-r, r}=\log \operatorname{Det}\left(Q_{1}+\sqrt{\Delta_{Y}}\right)+\log \operatorname{Det}\left(Q_{2}+\sqrt{\Delta_{Y}}\right)$.

Combining Corollary 4.2 with Corollary 4.7 and (4.2), (4.8), we complete the proof of Theorem 1.4.

\section{The adiabatic DeComposition of the ANALYTiC torsion}

In this section, we are going to prove Theorem 1.6 and Theorem 1.7. Recall that $M$ is a closed manifold of dimension $m$ with the product structure near a hypersurface $Y$. We define $M_{r}, M_{1, r}$ and $M_{2, r}$ as in Section 4 and suppose that $\rho_{M_{r}}\left(\rho_{M_{1, r}}\right.$, $\left.\rho_{M_{2, r}}, \rho_{Y}\right)$ is an orthogonal representation of $\pi_{1}\left(M_{r}\right)\left(\pi_{1}\left(M_{1, r}\right), \pi_{1}\left(M_{2, r}\right), \pi_{1}(Y)\right)$ to $S O(n)$, respectively. Then we can construct a flat bundle $E_{\rho_{M_{r}}}=\tilde{M}_{r} \times_{\rho_{M_{r}}} \mathbb{R}^{n}$, where $\tilde{M}_{r}$ is the universal cover of $M_{r}$. The flat bundles $E_{\rho_{M_{1, r}}}, E_{\rho_{M_{2, r}}}$ and $E_{\rho_{Y}}$ are defined in the same way.

For each $q$, denote by $\Delta_{M_{r}}^{q}:=\left(d_{q}+d_{q}^{*}\right)^{2}$ the Hodge Laplacian acting on $q$-forms valued in $E_{\rho_{M_{r}}}$. Then the analytic torsion $\tau\left(M_{r}, \rho_{M_{r}}\right)$ is defined by

$$
\log \tau\left(M_{r}, \rho_{M_{r}}\right)=\frac{1}{2} \sum_{q=0}^{m}(-1)^{q} \cdot q \cdot \log \operatorname{Det} \Delta_{M_{r}}^{q} .
$$

To define the analytic torsion on $M_{i, r}$, we choose the absolute or the relative boundary condition on $Y_{0}$. Near $Y_{0}$, a differential $q$-form $\omega$ can be expressed by

$$
\omega=\omega_{1}+d u \wedge \omega_{2},
$$

where $\omega_{1}$ and $\omega_{2}$ do not contain $d u$.

Definition 5.1. Suppose that a $q$-form $\omega$ in $M_{1, r}$ is expressed as in (5.1).

(1) $\omega$ satisfies the absolute boundary condition if $\left.\left(\partial_{u} \omega_{1}\right)\right|_{Y_{0}}=0$ and $\left.\omega_{2}\right|_{Y_{0}}=0$.

(2) $\omega$ satisfies the relative boundary condition if $\left.\omega_{1}\right|_{Y_{0}}=0$ and $\left.\left(\partial_{u} \omega_{2}\right)\right|_{Y_{0}}=0$.

We denote by $\Omega_{a b s}^{q}\left(M_{i, r}\right), \Omega_{r e l}^{q}\left(M_{i, r}\right)$ the sets of all $q$-forms valued in $E_{M_{i, r}}$ satisfying the absolute and the relative boundary conditions, respectively. We also denote by $\Delta_{M_{i, r}, a b s}^{q}, \Delta_{M_{i, r}, r e l}^{q}$ the Laplacian acting on $q$-forms valued in $E_{M_{i, r}}$ with

$$
\operatorname{Dom}\left(\Delta_{M_{i, r}, a b s}^{q}\right)=\Omega_{a b s}^{q}\left(M_{i, r}\right), \quad \operatorname{Dom}\left(\Delta_{M_{i, r}, r e l}^{q}\right)=\Omega_{r e l}^{q}\left(M_{i, r}\right) .
$$

Then the analytic torsions $\tau_{a b s}\left(M_{i, r}, \rho_{M_{i, r}}\right)$ and $\tau_{r e l}\left(M_{i, r}, \rho_{M_{i, r}}\right)$ are defined by

$$
\begin{aligned}
& \log \tau_{a b s}\left(M_{i, r}, \rho_{M_{i, r}}\right)=\frac{1}{2} \sum_{q=0}^{m}(-1)^{q} \cdot q \cdot \log \operatorname{Det} \Delta_{M_{i, r}, a b s}^{q}, \\
& \log \tau_{r e l}\left(M_{i, r}, \rho_{M_{i, r}}\right)=\frac{1}{2} \sum_{q=0}^{m}(-1)^{q} \cdot q \cdot \log \operatorname{Det} \Delta_{M_{i, r}, r e l}^{q} .
\end{aligned}
$$

It is a well-known fact (cf. [11]) that

$$
\operatorname{Ker} \Delta_{M_{i, r}, a b s}^{q} \cong H^{q}\left(M_{i, r} ; \rho_{M_{i, r}}\right), \quad \operatorname{Ker} \Delta_{M_{i, r}, r e l}^{q} \cong H^{q}\left(M_{i, r}, Y ; \rho_{M_{i, r}}\right) .
$$


We consider $M_{1, r}$ (a manifold with boundary $Y$ ) first. Recall that $M_{1, r}=$ $M_{1} \cup_{Y_{-r}} N_{-r, 0}$ with $N_{-r, 0}=[-r, 0] \times Y$, and $Y_{-r}=\{-r\} \times Y, Y_{0}=\{0\} \times Y$. We denote by $B, D$ the Dirichlet boundary conditions on $Y_{-r}, Y_{0}$, respectively.

For a given representation $\rho_{M_{1, r}}: \pi_{1}\left(M_{1, r}\right) \rightarrow S O(n)$, define $\rho_{Y}: \pi_{1}(Y) \rightarrow$ $S O(n)$ by $\rho_{Y}=\rho_{M_{1, r}} \circ \iota_{Y}$, where $\iota_{Y}: \pi_{1}(Y) \rightarrow \pi_{1}\left(M_{1, r}\right)$ is the natural homomorphism. Then the restriction of the bundle $E_{\rho_{M_{1, r}}}$ to $Y$ is isomorphic to $E_{\rho_{Y}},(c f$. [11]).

The set $\Omega^{q}\left(N_{-r, 0},\left.E_{\rho_{M_{1, r}}}\right|_{N_{-r, 0}}\right)$ of $q$-forms valued in $\left.E_{\rho_{M_{1, r}}}\right|_{N_{-r, 0}}$ can be decomposed as follows:

$$
\begin{aligned}
\Omega^{q}\left(N_{-r, 0},\left.E_{\rho_{M_{1, r}}}\right|_{N_{-r, 0}}\right) & =C^{\infty}\left([-r, 0],\left.E_{\rho_{M_{1, r}}}\right|_{N_{-r, 0}}\right) \otimes \Omega^{q}\left(Y, E_{\rho_{Y}}\right) \\
& \oplus d u \wedge C^{\infty}\left([-r, 0],\left.E_{\rho_{M_{1, r}}}\right|_{N_{-r, 0}}\right) \otimes \Omega^{q-1}\left(Y, E_{\rho_{Y}}\right) .
\end{aligned}
$$

From this decomposition, the Laplacian $\Delta_{M_{1, r}}^{q}$, when restricted to $N_{-r, 0}$, can be expressed as

$$
\Delta_{M_{1, r}}^{q}=-\partial_{u}^{2}+\left(\begin{array}{cc}
\Delta_{Y}^{q} & 0 \\
0 & \Delta_{Y}^{q-1}
\end{array}\right),
$$

where $\Delta_{Y}^{q}$ is the Laplacian acting on $q$-forms on $Y$, valued in $E_{\rho_{Y}}$. Here and throughout this section we use the convention that $\Delta_{Y}^{q}=0$ for $q<0$ or $q \geq m$.

To describe the gluing formula of the type of Theorem 1.1 (or Corollary 1.3) in this context, we need to define modified Dirichlet-to-Neumann operators $Q_{1}^{q}$, $Q_{N_{-r, 0}, a b s}^{q}$ and $Q_{N_{-r, 0}, r e l}^{q}$ as follows. For simplicity, set $E=\left(\bigwedge^{q} T^{*} M_{1, r}\right) \otimes E_{\rho_{M_{1, r}}}$. For a given $f \in C^{\infty}\left(\left.E\right|_{Y_{-r}}\right)$, choose smooth sections $\phi \in C^{\infty}\left(\left.E\right|_{M_{1}}\right), \psi_{a b s} \in$ $C^{\infty}\left(\left.E\right|_{N_{-r, 0}}\right)$ and $\psi_{r e l} \in C^{\infty}\left(\left.E\right|_{N_{-r, 0}}\right)$ such that

$$
\Delta_{M_{1}}^{q} \phi=0, \quad \Delta_{N_{-r, 0}}^{q} \psi_{a b s}=\Delta_{N_{-r, 0}}^{q} \psi_{r e l}=0,\left.\quad \phi\right|_{Y_{-r}}=\left.\psi_{a b s}\right|_{Y_{-r}}=\left.\psi_{r e l}\right|_{Y_{-r}}=f,
$$

and $\psi_{a b s}\left(\psi_{r e l}\right)$ satisfies the absolute (relative) boundary condition on $Y_{0}$, respectively. Then we define

$$
\begin{gathered}
Q_{1}^{q}(f)=\left.\left(\partial_{u} \phi\right)\right|_{Y_{-r}}, \\
Q_{N_{-r, 0}, a b s}^{q}(f)=\left.\left(-\partial_{u} \psi_{a b s}\right)\right|_{Y_{-r}}, \quad Q_{N_{-r, 0}, r e l}^{q}(f)=\left.\left(-\partial_{u} \psi_{r e l}\right)\right|_{Y_{-r}},
\end{gathered}
$$

and

$$
R_{B, a b s}^{q}=Q_{1}^{q}+Q_{N_{-r, 0}, a b s}^{q}, \quad R_{B, r e l}^{q}=Q_{1}^{q}+Q_{N_{-r, 0}, r e l}^{q} .
$$

Then the following theorem can be proved in the same way as Theorem 1.1 ( $c f$. the Remark after Corollary 1.3).

Theorem 5.2. We denote $k_{q}=\operatorname{dimKer} \Delta_{Y}^{q}$. Then:

$$
\begin{gathered}
\text { (1) } \log \operatorname{Det} \Delta_{M_{1, r}, D}^{q}-\log \operatorname{Det} \Delta_{M_{1}, B}^{q}-\log \operatorname{Det} \Delta_{N_{-r, 0}, B, D}^{q} \\
=-\log 2\left(\zeta_{\Delta_{Y}^{q-1}}(0)+\zeta_{\Delta_{Y}^{q}}(0)+k_{q-1}+k_{q}\right)+\log \operatorname{Det} R_{B, D}^{q} . \\
\text { (2) } \log \operatorname{Det} \Delta_{M_{1, r}, a b s}^{q}-\log \operatorname{Det} \Delta_{M_{1}, B}^{q}-\log \operatorname{Det} \Delta_{N_{-r, 0}, B, a b s}^{q} \\
=-\log 2\left(\zeta_{\Delta_{Y}^{q-1}}(0)+\zeta_{\Delta_{Y}^{q}}(0)+k_{q-1}+k_{q}\right)+\log \operatorname{Det} R_{B, a b s}^{q} . \\
\text { (3) } \log \operatorname{Det} \Delta_{M_{1, r}, r e l}^{q}-\log \operatorname{Det} \Delta_{M_{1}, B}^{q}-\log \operatorname{Det} \Delta_{N_{-r, 0}, B, r e l}^{q} \\
=-\log 2\left(\zeta_{\Delta_{Y}^{q-1}}(0)+\zeta_{\Delta_{Y}^{q}}(0)+k_{q-1}+k_{q}\right)+\log \operatorname{Det} R_{B, r e l}^{q} .
\end{gathered}
$$


We next describe the operators $\Delta_{N_{-r, 0}, B, a b s}^{q}$ and $\Delta_{N_{-r, 0}, B, r e l}^{q}$. From the decomposition (5.2), we have

$$
\begin{aligned}
& \Delta_{N_{-r, 0}, B, a b s}^{q}=\left(\begin{array}{cc}
\left(-\partial_{u}^{2}+\Delta_{Y}^{q}\right)_{N_{-r, 0}, B, C} & 0 \\
0 & \left(-\partial_{u}^{2}+\Delta_{Y}^{q-1}\right)_{N_{-r, 0}, B, D}
\end{array}\right), \\
& \Delta_{N_{-r, 0}, B, r e l}^{q}=\left(\begin{array}{cc}
\left(-\partial_{u}^{2}+\Delta_{Y}^{q}\right)_{N_{-r, 0}, B, D} & 0 \\
0 & \left(-\partial_{u}^{2}+\Delta_{Y}^{q-1}\right)_{N_{-r, 0}, B, C}
\end{array}\right),
\end{aligned}
$$

where $C$ means the Neumann boundary condition on $Y_{0}$ and $B(D)$ means the Dirichlet boundary condition on $Y_{-r}\left(Y_{0}\right)$. Hence, we have

$$
\begin{aligned}
& \log \operatorname{Det} \Delta_{N_{-r, 0}, B, a b s}^{q}-\log \operatorname{Det} \Delta_{N_{-r, 0}, B, D}^{q} \\
& \quad=\log \operatorname{Det}\left(-\partial_{u}^{2}+\Delta_{Y}^{q}\right)_{N_{-r, 0}, B, C}-\log \operatorname{Det}\left(-\partial_{u}^{2}+\Delta_{Y}^{q}\right)_{N_{-r, 0}, B, D}, \\
& \log \operatorname{Det} \Delta_{N_{-r, 0}, B, r e l}^{q}-\log \operatorname{Det} \Delta_{N_{-r, 0}, B, D}^{q} \\
& \quad=\log \operatorname{Det}\left(-\partial_{u}^{2}+\Delta_{Y}^{q-1}\right)_{N_{-r, 0}, B, C}-\log \operatorname{Det}\left(-\partial_{u}^{2}+\Delta_{Y}^{q-1}\right)_{N_{-r, 0}, B, D} .
\end{aligned}
$$

Now we assume that $Q_{1}^{q}+\left(\begin{array}{cc}\sqrt{\Delta_{Y}^{q}} & 0 \\ 0 & \sqrt{\Delta_{Y}^{q-1}}\end{array}\right)$ is invertible. Since the Hodge Laplacian $\Delta_{M_{r}}^{q}$ is a Dirac Laplacian satisfying (1.1), by Corollary 4.5 we have $k_{q-1}=$ $k_{q}=0$ ( $c f$. the Remark below Theorem 1.6). By Corollary 1.5 and (5.4), (5.5) we have

$$
\begin{aligned}
& \lim _{r \rightarrow \infty}\left\{\log \operatorname{Det} \Delta_{N_{-r, 0}, B, a b s}^{q}-\log \operatorname{Det} \Delta_{N_{-r, 0}, B, D}^{q}\right\}=\frac{1}{2} \log \operatorname{Det} \Delta_{Y}^{q}, \\
& \lim _{r \rightarrow \infty}\left\{\log \operatorname{Det} \Delta_{N_{-r, 0}, B, r e l}^{q}-\log \operatorname{Det} \Delta_{N_{-r, 0}, B, D}^{q}\right\}=\frac{1}{2} \log \operatorname{Det} \Delta_{Y}^{q-1} .
\end{aligned}
$$

From Theorem 5.2 we have

$$
\begin{aligned}
\log \operatorname{Det} \Delta_{M_{1, r}, a b s}^{q}-\log \operatorname{Det} \Delta_{M_{1, r}, D}^{q} \\
=\left(\log \operatorname{Det} \Delta_{N_{-r, 0}, B, a b s}^{q}-\log \operatorname{Det} \Delta_{N_{-r, 0}, B, D}^{q}\right) \\
\quad+\log \operatorname{Det} R_{B, a b s}^{q}-\log \operatorname{Det} R_{B, D}^{q}
\end{aligned}
$$

and

$$
\begin{aligned}
\log \operatorname{Det} \Delta_{M_{1, r}, r e l}^{q}-\log \operatorname{Det} \Delta_{M_{1, r}, D}^{q} \\
=\left(\log \operatorname{Det} \Delta_{N_{-r, 0}, B, r e l}^{q}-\log \operatorname{Det} \Delta_{N_{-r, 0}, B, D}^{q}\right) \\
\quad+\log \operatorname{Det} R_{B, r e l}^{q}-\log \operatorname{Det} R_{B, D}^{q} .
\end{aligned}
$$

Lemma 5.3. Suppose that $Q_{1}^{q}+\left(\begin{array}{cc}\sqrt{\Delta_{Y}^{q}} & 0 \\ 0 & \sqrt{\Delta_{Y}^{q-1}}\end{array}\right)$ is invertible. Then

$$
\begin{gathered}
\lim _{r \rightarrow \infty} \log \operatorname{Det} R_{B, a b s}^{q}=\lim _{r \rightarrow \infty} \log \operatorname{Det} R_{B, r e l}^{q}=\lim _{r \rightarrow \infty} \log \operatorname{Det} R_{B, D}^{q} \\
=\log \operatorname{Det}\left(Q_{1}^{q}+\left(\begin{array}{cc}
\sqrt{\Delta_{Y}^{q}} & 0 \\
0 & \sqrt{\Delta_{Y}^{q-1}}
\end{array}\right)\right) .
\end{gathered}
$$

Proof. The last equality is exactly the assertion (1) in Corollary 4.7. We are going to show that

$$
\lim _{r \rightarrow \infty} \log \operatorname{Det} R_{B, a b s}^{q}=\log \operatorname{Det}\left(Q_{1}^{q}+\left(\begin{array}{cc}
\sqrt{\Delta_{Y}^{q}} & 0 \\
0 & \sqrt{\Delta_{Y}^{q-1}}
\end{array}\right)\right) .
$$


The case of $\log \operatorname{Det} R_{B, \text { rel }}^{q}$ can be proved in the same way.

By a direct computation one can check the following. For $f \in \Omega^{q}\left(Y, E_{\rho_{Y}}\right)$ with $\Delta_{Y}^{q} f=\lambda f$,

$$
R_{B, a b s}^{q}(f)=Q_{1}^{q}(f)+\left(\sqrt{\lambda}-\frac{2 \sqrt{\lambda} e^{-\sqrt{\lambda} r}}{e^{\sqrt{\lambda} r}+e^{-\sqrt{\lambda} r}}\right) f .
$$

For $g \in \Omega^{q-1}\left(Y, E_{\rho_{Y}}\right)$ with $\Delta_{Y}^{q-1} g=\mu g$,

$$
R_{B, a b s}^{q}(d u \wedge g)=Q_{1}^{q}(d u \wedge g)+\left(\sqrt{\mu}+\frac{2 \sqrt{\mu} e^{-\sqrt{\mu} r}}{e^{\sqrt{\mu} r}-e^{-\sqrt{\mu} r}}\right) d u \wedge g .
$$

Then the result follows from Lemma 4.1 .

From (5.6)-(5.9) and Lemma 5.3, we have the following corollary.

Corollary 5.4. Suppose that $Q_{1}^{q}+\left(\begin{array}{cc}\sqrt{\Delta_{Y}^{q}} & 0 \\ 0 & \sqrt{\Delta_{Y}^{q-1}}\end{array}\right)$ is invertible for each $q$. Then the following equalities hold:

$$
\begin{gathered}
\lim _{r \rightarrow \infty}\left\{\log \operatorname{Det} \Delta_{M_{1, r}, a b s}^{q}-\log \operatorname{Det} \Delta_{M_{1, r}, D}^{q}\right\} \\
= \begin{cases}\frac{1}{2} \log \operatorname{Det} \Delta_{Y}^{q} & (0 \leq q \leq m-1), \\
0 & (q=m) .\end{cases} \\
\lim _{r \rightarrow \infty}\left\{\begin{array}{ll}
\log \operatorname{Det} \Delta_{M_{1, r}, r e l}^{q}-\log \operatorname{Det} \Delta_{M_{1, r}, D}^{q}
\end{array}\right\} \\
= \begin{cases}\frac{1}{2} \log \operatorname{Det} \Delta_{Y}^{q-1} & (1 \leq q \leq m), \\
0 & (q=0) .\end{cases}
\end{gathered}
$$

Now we are ready to prove Theorem 1.6. We have

$$
\begin{aligned}
\lim _{r \rightarrow \infty}\{ & \left.\log \tau_{a b s}\left(M_{1, r}, \rho_{M_{1, r}}\right)-\log \tau_{r e l}\left(M_{1, r}, \rho_{M_{1, r}}\right)\right\} \\
= & \lim _{r \rightarrow \infty} \frac{1}{2} \sum_{q=0}^{m}(-1)^{q} \cdot q \cdot\left(\log \operatorname{Det} \Delta_{M_{1, r}, a b s}-\log \operatorname{Det} \Delta_{M_{1, r}, D}\right) \\
& -\lim _{r \rightarrow \infty} \frac{1}{2} \sum_{q=0}^{m}(-1)^{q} \cdot q \cdot\left(\log \operatorname{Det} \Delta_{M_{1, r}, r e l}-\log \operatorname{Det} \Delta_{M_{1, r}, D}\right) \\
= & \frac{1}{4} \sum_{q=0}^{m-1}(-1)^{q} \cdot q \cdot \log \operatorname{Det}\left(\Delta_{Y}^{q}\right)-\frac{1}{4} \sum_{q=1}^{m}(-1)^{q} \cdot q \cdot \log \operatorname{Det}\left(\Delta_{Y}^{q-1}\right) \\
= & \frac{1}{2} \sum_{q=0}^{m-1}(-1)^{q} \cdot q \cdot \log \operatorname{Det}\left(\Delta_{Y}^{q}\right)+\frac{1}{4} \sum_{q=0}^{m-1}(-1)^{q} \cdot \log \operatorname{Det}\left(\Delta_{Y}^{q}\right) \\
= & \tau\left(Y, \rho_{Y}\right) .
\end{aligned}
$$

This completes the proof of Theorem 1.6.

Next, we take care of the closed manifold $M_{r}=M_{1, r} \cup_{Y_{0}} M_{2, r}$. From Theorem 1.4, we have

$$
\begin{gathered}
\lim _{r \rightarrow \infty}\left\{\log \operatorname{Det} \Delta_{M_{r}}^{q}-\log \operatorname{Det} \Delta_{M_{1, r}, D}^{q}-\log \operatorname{Det} \Delta_{M_{2, r}, D}^{q}\right\} \\
=\frac{1}{2}\left(\log \operatorname{Det} \Delta_{Y}^{q}+\log \operatorname{Det} \Delta_{Y}^{q-1}\right) .
\end{gathered}
$$


On the other hand,

$$
\begin{aligned}
& \lim _{r \rightarrow \infty}\{\left.\log \operatorname{Det} \Delta_{M_{r}}^{q}-\log \operatorname{Det} \Delta_{M_{1, r}, D}^{q}-\log \operatorname{Det} \Delta_{M_{2, r}, D}^{q}\right\} \\
&=\lim _{r \rightarrow \infty}\{(\left.\log \operatorname{Det} \Delta_{M_{r}}^{q}-\log \operatorname{Det} \Delta_{M_{1, r}, a b s}^{q}-\log \operatorname{Det} \Delta_{M_{2, r}, r e l}^{q}\right) \\
&+\left(\log \operatorname{Det} \Delta_{M_{1, r}, a b s}^{q}-\log \operatorname{Det} \Delta_{M_{1, r}, D}^{q}\right) \\
&\left.+\left(\log \operatorname{Det} \Delta_{M_{2, r}, r e l}^{q}-\log \operatorname{Det} \Delta_{M_{2, r}, D}^{q}\right)\right\} .
\end{aligned}
$$

From Corollary 5.4 we have

$$
\lim _{r \rightarrow \infty}\left\{\log \operatorname{Det} \Delta_{M_{r}}^{q}-\log \operatorname{Det} \Delta_{M_{1, r}, a b s}^{q}-\log \operatorname{Det} \Delta_{M_{2, r}, r e l}^{q}\right\}=0,
$$

and therefore we obtain

$$
\lim _{r \rightarrow \infty}\left\{\tau\left(M_{r}, \rho_{M_{r}}\right)-\tau_{a b s}\left(M_{1, r}, \rho_{M_{1, r}}\right)-\tau_{r e l}\left(M_{2, r}, \rho_{M_{2, r}}\right)\right\}=0,
$$

which completes the proof of Theorem 1.7.

\section{REFERENCES}

[1] M. F. Atiyah, V. K. Patodi and I. M. Singer, Spectral asymmetry and Riemannian geometry I, Math. Proc. Camb. Phil. Soc. 77 (1975), 43-69. MR 53:1655a

[2] B. Booß-Bavnbek, and K.P. Wojciechowski, Elliptic Boundary Problems for Dirac Operators, Birkhäuser, Boston, 1993. MR 94h:58168

[3] D. Burghelea, L. Friedlander and T. Kappeler, Mayer-Vietoris type formula for determinants of elliptic differential operators, J. of Funct. Anal. 107 (1992), 34-66. MR 93f:58242

[4] D. Burghelea, L. Friedlander, T. Kappeler and P. McDonald, Analytic and Reidemeister torsion for representations in finite type Hilbert modules, Geom. and Funct. Anal. 6 (1996), 751-859. MR 97i:58177

[5] S. Cappell, R. Lee and E. Miller, Self-adjoint elliptic operators and manifold decompositions, Part I: Low eigenmodes and stretching, Comm. Pure Appl. Math. 96 (1996), 825-866. MR 97g:58162

[6] S. Klimek, K.P. Wojciechowski, Adiabatic cobordism theorems for analytic torsion and $\eta$ invariant, J. of Funct. Anal. 136 (1996), 269-293. MR 97a:58195

[7] Y. Lee, Mayer-Vietoris formula for the determinant of a Laplace operator on an even dimensional manifold, Proc. Amer. Math. Soc. 121-6 (1995), 1933-1940. MR 95g:58255

[8] Y. Lee, Mayer-Vietoris formula for the determinants of elliptic operators of Laplace-Beltrami type (after Burghelea, Friedlander and Kappeler), Diff. Geom. and Its Appl. 7 (1997), 325340. MR 99a:58163

[9] Y. Lee, Burghelea-Friedlander-Kappeler's gluing formula and the adiabatic decomposition of the zeta-determinant of a Dirac Laplacian, to appear in Manuscripta Math.

[10] P. Park and K. Wojciechowski with Appendix by Y. Lee, Adiabatic decomposition of the $\zeta$ determinant of the Dirac Laplacian I. The case of an invertible tangential operator, Comm. in PDE. 27 (2002), 1407-1435.

[11] D.B. Ray and I.M. Singer, R-torsion and the Laplacian on Riemannian manifolds, Adv. in Math. 7 (1971), 145-209. MR 45:4447

[12] A. Voros, Spectral function, special functions and Selberg zeta function, Comm. Math. Phys. 110 (1987), 439-465. MR 89b:58173

Department of Mathematics, Inha University, Inchon, 402-751, Korea

E-mail address: ywlee@math.inha.ac.kr 